\title{
Treinamento Aeróbio em Natação Melhora a Resposta de Parâmetros Metabólicos de Ratos Durante Teste de Esforço
}

\author{
Aerobic Swimming Training Improves Metabolic Parameters Response \\ During Exertion Test in Rats
}

CIÊNCIAS DO EXERCÍCIO E DO ESPORTE

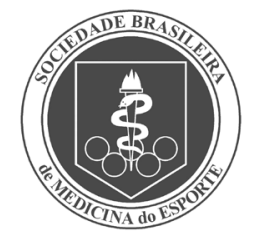

Artigo Original
Juliana Silveira Freitas'

Miguel Araújo Carneiro-Junior²

Frederico Souzalima Caldoncelli

Franco $^{3}$

Levy Silva Rezende

Andreza Soares dos Santos

Hugo de Oliveira Maia'

João Carlos Bouzas Marins'

Antônio José Natali'

1. Departamento de Educação Física da Universidade Federal de Viçosa Viçosa/MG;

2. Programa de Pós-Graduação em Ciências Fisiológicas da Universidade Federal do Espírito

Santo - Vitória/ES;

3. Instituto Federal de Educação Tecnológica Sudeste de Minas Gerais - Rio Pomba/MG.

Endereço para correspondência: Prof. PhD. Antônio José Natali Departamento de Educação Física U.F. Viçosa

Avenida P.H. Rolfs, s/n 36570-000 - Viçosa, MG

E-mail: anatali@ufv.br

\section{RESUMO}

Foram investigados os efeitos do treinamento aeróbio em natação com baixa intensidade sobre as respostas do lactato e da glicose sanguíneos de ratos durante teste de esforço. Ratos Wistar adultos foram distribuídos aleatoriamente em dois grupos: sedentário $(n=6)$ e treinado $(n=6)$. Todos receberam água e ração ad libitum e foram mantidos em ambiente com temperatura de $22 \pm 2^{\circ} \mathrm{C}$ e ciclo claro/escuro de 12 horas. O grupo treinado foi submetido a um programa de natação contínua sem sobrecarga, 30 min/dia, cinco dias/semana, por seis semanas. Três dias após a última sessão de treino, as concentrações sanguíneas de lactato e glicose foram medidas em três momentos durante dois testes de esforço de 20 minutos (repouso, 10 min e $20 \mathrm{~min}$ ), sendo um sem carga e outro com carga (5\% do peso corporal), separados por dois dias. Observou-se correlação inversa entre lactato e glicose durante o exercício ( $\rho=$ $-0,74 ; P<0,001)$. A concentração de lactato elevou-se do repouso para 10 min $(P<0,05)$ e estabilizouse entre 10 e 20 min, em ambos os grupos nos dois testes. No teste com carga, o lactato estabilizou-se em níveis mais elevados frente aos níveis sem carga $(P<0,05)$, nos dois grupos. Os animais treinados exibiram níveis de lactato mais baixos do que os sedentários $(P<0,05)$ nos dois testes. A glicose sanguínea decaiu do repouso até 20 min nos sedentários, no teste com carga $(P<0,05)$. Nos treinados, a glicose sanguínea estabilizou-se em ambos os testes $(P>0,05)$. Conclui-se que o treinamento aeróbio em natação aplicado foi capaz de alterar as respostas do lactato e glicose sanguíneos de ratos durante os testes de esforço.

Palavras-chave: atividade física, limiar anaeróbio, lactato, glicose.

\section{ABSTRACT}

The effects of low intensity aerobic swimming training on blood lactate and glucose responses in rats were investigated during exertion test. Twelve adult male Wistar rats were randomly divided into two groups: sedentary $(n=6)$ and trained $(n=6)$. All animals received water and food ad libitum and were kept in a room with temperature of $22 \pm 2^{\circ} \mathrm{C}$ and dark/light cycle of 12 hours. Animals from trained group were submitted to a swimming training protocol of $30 \mathrm{~min} /$ day, 5 days/week, for 6 weeks. Sedentary animals did not exercise. Three days after the last training session all animals were submitted to two 20-minute swimming tests with 48 hour-interval, being one unloaded and the other with a load of $5 \%$ of body weight. Blood lactate and glucose were measured at rest, $10 \mathrm{~min}$ and $20 \mathrm{~min}$ of exercise. Negative correlation between blood lactate and glucose levels was observed during the exertion tests $(\rho=-0.74, P<0.001)$. Blood lactate concentration increased from rest to $10 \mathrm{~min}$ of exercise and stabilized from 10 to $20 \mathrm{~min}$ of exercise in both exercised and sedentary animals $(P<0.05)$ during the unloaded exertion test. Blood lactate stabilized at higher levels when compared to those in the unloaded test in both groups $(P<0.05)$ during the loaded exertion test. Trained rats presented lower levels of blood lactate than sedentary animals in both exercise tests $(P<0.05)$. Blood glucose declined from rest to 10 min of exercise in sedentary rats during the loaded test $(P<0.05)$. However, in trained animals blood glucose stabilized in both exercise tests $(P>0.05)$. It was concluded that aerobic swimming training changed blood lactate and glucose response in rats during exertion test.

Keywords: physical activity, anaerobic threshold, lactate, glucose. 


\section{INTRODUÇÃO}

O limiar de lactato é considerado um dos principais parâmetros para determinação do limiar anaeróbio(1). Embora ainda existam divergências a respeito do mecanismo de controle do lactato durante a realização de exercícios, a resposta metabólica da conversão da glicose em lactato sanguíneo desempenha um importante papel no controle das variáveis do treinamento. O limiar de lactato é um parâmetro utilizado para prescrição da intensidade de esforço, bem como para acompanhar os efeitos do treinamento e as alterações induzidas pelo exercício, tanto em humanos ${ }^{(1)} \operatorname{como~em~ratos~}^{(2)}$.

Durante a realização de exercícios com intensidades progressivas (moderadas a altas), a oferta de oxigênio passa a não suprir seu requerimento, ocorre aumento acentuado das demandas energéticas, que passam a ser obtidas através de mecanismos anaeróbios pela conversão da glicose em ácido pirúvico e, posteriormente, em ácido lático ${ }^{(3,4)}$. Se o exercício for mantido, a produção de lactato excede sua remoção e o lactato se acumula no organismo.

O equilíbrio entre a velocidade de produção de lactato e a velocidade de sua remoção do sangue é conhecido como limiar de lactato $^{(5,6)}$. Esse equilíbrio ocorre geralmente numa concentração de $4,0 \mathrm{mmol} / \mathrm{L}^{(7,8)}$. Espera-se que cargas correspondentes a essa concentração de lactato possam ser suportadas durante um teste prolongado de carga constante, constituindo-se como um referencial importante na avaliação e controle do treinamento aeróbio ${ }^{(9)}$.

Gobatto et al. (2) demonstraram, em um estudo com ratos submetidos a um programa de natação de alta intensidade, que o treinamento não alterou o valor do limiar de lactato $(5,5 \mathrm{mmol} / \mathrm{L})$, porém, este foi atingido numa intensidade maior de exercício (5\% do peso corporal antes e $8 \%$ após o período de treinamento). Cunha et al. ${ }^{(10)}$ treinaram ratos idosos em natação com intensidade no limiar de lactato (sobrecarga de $5 \%$ do peso corporal) e, também, mostraram que houve aumento na intensidade do exercício para atingir o limiar de lactato. Entretanto, pouco se sabe sobre os efeitos do treinamento aeróbio em natação com baixa intensidade (sem sobrecarga) sobre a concentração de lactato sanguíneo em ratos.

Em adição ao glicogênio hepático, o glicogênio muscular é um combustível essencial durante o exercício físico, embora somente as células musculares possam utilizá-lo como fonte de energia. Os exercícios físicos são estímulos que resultam na depleção dos estoques de glicogênio muscular ${ }^{(11)}$; assim, a desaceleração da taxa de utilização do glicogênio é importante para a realização do exercício aeróbio de longa duração, sendo o glicogênio muscular um fator limitante para a manutenção do exercício(12). O aumento da demanda de energia promovida pelo exercício provoca maior utilização da glicose pelos tecidos e órgãos e, consequentemente, sua diminuição em níveis plasmáticos. Acredita-se que, a partir de determinado momento de atividade, catecolaminas e glicocorticoides possam estimular a glicogenólise hepática, como estratégia fisiológica de manutenção da glicemia sanguínea ${ }^{(11)}$.

Segundo Silveira et al. ${ }^{(13)}$, o exercício contínuo realizado em intensidade submáxima pode induzir um efeito inibitório na via glicolítica e aumentar a produção de energia pela via oxidativa, o que promoveria diminuição da utilização do glicogênio e produção de lactato, aumento da oxidação de lipídios e manutenção da glicemia sanguínea. Além disso, considera-se que o exercício moderado promova o equilíbrio na taxa de mobilização e utilização da glicose como substrato energético, mantendo a glicemia sanguínea em níveis normais ${ }^{(11)}$. Todavia, o comportamento da glicemia sanguínea de ratos submetidos ao treinamento aeróbio em natação com baixa intensidade (sem sobrecarga) não está bem esclarecido.
Dessa forma, o que se questiona é se haveria a necessidade de aplicação do treinamento de natação com alta intensidade, acima do limiar anaeróbio(2), e/ou na intensidade correspondente ao limiar anaeróbio(10) para melhorar a capacidade aeróbia de ratos, ou se o treinamento em natação com baixa intensidade (sem sobrecarga) seria suficiente para melhorar a capacidade aeróbia de ratos. Adicionalmente, qual seria o comportamento da glicemia sanguínea em ratos submetidos ao treinamento em natação com baixa intensidade (sem sobrecarga)?

Assim, o objetivo deste trabalho foi investigar os efeitos de um programa de treinamento aeróbio em natação com baixa intensidade (sem sobrecarga) sobre a resposta do lactato e da glicose sanguínea em ratos durante teste de esforço.

\section{METODOLOGIA}

\section{Animais de experimentação e tratamento}

Doze ratos Wistar machos, com idade de 70 dias e peso corporal de 192,92 \pm 14,63g (média \pm DP) foram utilizados. Os animais foram alocados aleatoriamente em dois grupos: sedentário $(n=6)$ e treinado $(n=6)$, sendo alojados em gaiolas coletivas (seis por gaiola). Os animais receberam ração comercial $\left(\right.$ Soci $\left.^{\circledR}\right)$ e água ad libitum, foram mantidos em regime de luminosidade de 12 horas claro/escuro em sala com temperatura de $22 \pm 2^{\circ} \mathrm{C}$. Os animais foram obtidos no Biotério Central do Centro de Ciências Biológicas e da Saúde, da Universidade Federal de Viçosa, e todas as "normas de cuidados para experimentação com animais" foram seguidas, conforme o Colégio Brasileiro de Experimentação Animal (Cobea).

\section{Protocolo de treinamento}

Na primeira semana de experimento, os animais do grupo treinado foram colocados em um tanque de alvenaria azulejado (largura: $65 \mathrm{~cm}$, comprimento: $75 \mathrm{~cm}$ e altura: $85 \mathrm{~cm})$ com água $\left(230^{\circ} \mathrm{C} \pm 1{ }^{\circ} \mathrm{C}\right)$ na profundidade de $45 \mathrm{~cm}$, para adaptação à temperatura da água e ao programa de exercício. A adaptação ao treinamento consistiu em natação sem sobrecarga com tempo progressivo durante cinco dias. No primeiro dia o tempo de exercício foi de 10 minutos, sendo aumentado em cinco minutos por dia, chegando aos 30 minutos no último dia da semana. Após a semana de adaptação iniciou-se o protocolo de treinamento com sessões diárias de 30 minutos, cinco dias por semana, por seis semanas. Esse treinamento é considerado aeróbio, pois os animais nadaram sem sobrecarga adicional, o que representa intensidade abaixo daquela correspondente ao limiar anaeróbio de ratos Wistar ${ }^{(2,10)}$.

\section{Determinação das concentrações de lactato e glicose san- guínea}

Três dias após a última sessão de treinamento, os ratos foram submetidos individualmente a dois testes de esforço para determinação das concentrações sanguíneas de lactato e glicose, com intervalo de 48 horas entre os testes ${ }^{(2)}$. No primeiro teste, os animais nadaram por 20 minutos continuamente, sem sobrecarga, e as amostras de sangue foram coletadas em três momentos: em repouso (tempo 0), após 10 minutos de esforço (tempo 10 min) e após o teste (tempo 20 min). Amostras sanguíneas (uma gota) foram coletadas da extremidade distal da cauda. No segundo teste, os animais nadaram por 20 minutos continuamente, com sobrecarga de $5 \%$ do peso corporal, e as amostras de sangue foram coletadas da mesma forma que no primeiro teste. Para evitar a diluição da amostra sanguínea com a água do tanque, a cauda do animal foi secada antes da coleta. As concentrações sanguíneas de lactato e glicose foram determinadas utilizando-se os analisadores portáteis de lactato (Accusport BM-Lactate) e glicose (Accu-Chek Advantage ${ }^{\circ}$ ) ambos da Roche Diagnostics (Mannheim, Alemanha). 


\section{Análise estatística}

As médias de lactato e glicose sanguíneos foram avaliadas por: ANOVA one-way de medidas repetidas entre os momentos do mesmo grupo, teste $t$ não pareado entre grupos em cada momento, teste $t$ pareado entre os testes de esforço sem e com sobrecarga, em cada grupo, e correlação de Pearson para relação entre concentração de lactato e glicose. Para as análises de múltiplas comparações post hoc foi utilizado o teste de Tukey em análises paramétricas e Dunn's em análises não-paramétricas. Utilizou-se o software Sigma Stat versão 3.0 (SPSS) para as análises estatísticas, empregando o nível de significância estatística de $\mathrm{P}<0,05$.

\section{RESULTADOS}

Não foi observada diferença $(P>0,05)$ entre os grupos no peso corporal inicial (sedentário: 194,7 $\pm 3,8$ e treinado: 192,2 \pm 8,3g; média \pm EPM) e peso corporal final (sedentário: 321,3 \pm 14,5 e treinado: 313,6 $\pm 9,0 \mathrm{~g})$.

A figura 1 apresenta os resultados no teste sem carga dos grupos sedentário e treinado. Nos animais sedentários a concentração de lactato elevou-se do repouso $(3,0 \pm 0,1 \mathrm{mmol} / \mathrm{L}$, média \pm EPM) para os tempos $10 \mathrm{~min}(6,1 \pm 0,9 \mathrm{mmol} / \mathrm{L} ; \mathrm{P}=0,005)$ e $20 \mathrm{~min}(5,6 \pm 0,5$ $\mathrm{mmol} / \mathrm{L} ; \mathrm{P}=0,014)$. Porém, não houve diferença no lactato entre os tempos $10 \mathrm{~min}$ e $20 \mathrm{~min}$. Nos animais treinados observou-se que 0 lactato sanguíneo também se elevou do repouso $(2,7 \pm 0,2 \mathrm{mmol} / \mathrm{L})$ para os tempos $10 \mathrm{~min}(3,8 \pm 0,4 \mathrm{mmol} / \mathrm{L} ; \mathrm{P}=0,014)$ e $20 \mathrm{~min}(3,6 \pm$ $0,2 \mathrm{mmol} / L ; P=0,029)$. Não houve diferença $(P>0,05)$ entre os tempos 10 e 20 min.

Ao comparar os animais treinados com os sedentários, observou-se que no repouso não houve diferença entre os grupos. Todavia, observou-se que o lactato sanguíneo na qual ocorreu a estabilização durante o teste de esforço sem carga foi inferior no grupo treinado (tempo 10 min: $3,8 \pm 0,4$ vs. $6,1 \pm 0,9 \mathrm{mmol} / \mathrm{L} ; P=0,039$ e tempo $20 \mathrm{~min}: 3,7 \pm 0,2$ vs. $5,6 \pm 0,6 \mathrm{mmol} / \mathrm{L} ; \mathrm{P}=0,006$; respectivamente).

Os resultados da glicose sanguínea dos animais sedentários mostraram redução significativa do repouso $(92,7 \pm 2,9 \mathrm{mg} / \mathrm{dL})$ para os tempos $10 \mathrm{~min}(78,5 \pm 3,1 \mathrm{~m} / \mathrm{dL} ; \mathrm{P}<0,05)$ e $20 \mathrm{~min}(79,2 \pm 4,1 \mathrm{mg} / \mathrm{dL}$; $P<0,05)$. Porém, entre os tempos 10 e 20 min, não houve diferença. Nos animais treinados a glicose sanguínea não foi diferente entre os tempos dos testes sem carga (repouso: 88,0 $\pm 3,4$; tempo 10: 80,3 \pm 1,0 e 20 minutos: $92,2 \pm 3,5 \mathrm{mg} / \mathrm{dL}$ ). Porém, verificou-se elevação entre os tempos 10 e 20 min $(P=0,029)$.

Entre os grupos sedentário e treinado, não houve alteração da glicose no repouso $(92,7 \pm 2,9 \mathrm{vs}$. $88,0 \pm 3,4 \mathrm{mg} / \mathrm{dL}$, respectivamente) e no tempo $10 \mathrm{~min}(78,5 \pm 3,1 \mathrm{vs} .80,3 \pm 1,0 \mathrm{mg} / \mathrm{dL}$, respectivamente). Entretanto, verificou-se que a glicose sanguínea no tempo 20 apresentou-se maior no grupo treinado do que no sedentário $(92,2 \pm 3,5$ vs. $79,2 \pm$ $4,1 \mathrm{mg} / \mathrm{dL}$; respectivamente; $P=0,037$ ).

A figura 2 apresenta os resultados no teste com carga dos grupos sedentário e treinado. A concentração de lactato elevou-se nos animais sedentários do repouso $(2,8 \pm 0,1 \mathrm{mmol} / \mathrm{L} ;$ média \pm EPM) para os tempos $10 \mathrm{~min}(7,1 \pm 0,7 \mathrm{mmol} / \mathrm{L}, \mathrm{P}<0,001)$ e $20 \mathrm{~min}(9,2 \pm 0,9 \mathrm{mmol} / \mathrm{L}, \mathrm{P}=$ 0,003). Porém, não houve diferença entre os tempos 10 min e 20 min. Nos animais treinados observou-se que o lactato sanguíneo também se elevou do repouso $(2,4 \pm 0,1 \mathrm{mmol} / \mathrm{L})$ para os tempos $10 \mathrm{~min}(6,2$ $\pm 0,6 \mathrm{mmol} / \mathrm{L}, \mathrm{P}<0,001)$ e $20 \mathrm{~min}(5,7 \pm 0,5 \mathrm{mmol} / \mathrm{L}, \mathrm{P}<0,001)$. Não houve diferença $(P>0,05)$ entre os tempos 10 e 20 min.

Nas comparações dos resultados entre os grupos sedentário e treinado, no teste com carga, verificou-se que em repouso $(2,4 \pm 0,1$ vs. 2,8 $\pm 0,1 \mathrm{mmol} / \mathrm{L}$, respectivamente) e $10 \mathrm{~min}(6,2 \pm 0,6 \mathrm{vs} .7,1 \pm 0,7 \mathrm{mmol} / \mathrm{L}$, respectivamente) não houve diferença $(P>0,05)$. Entretanto, notou-se

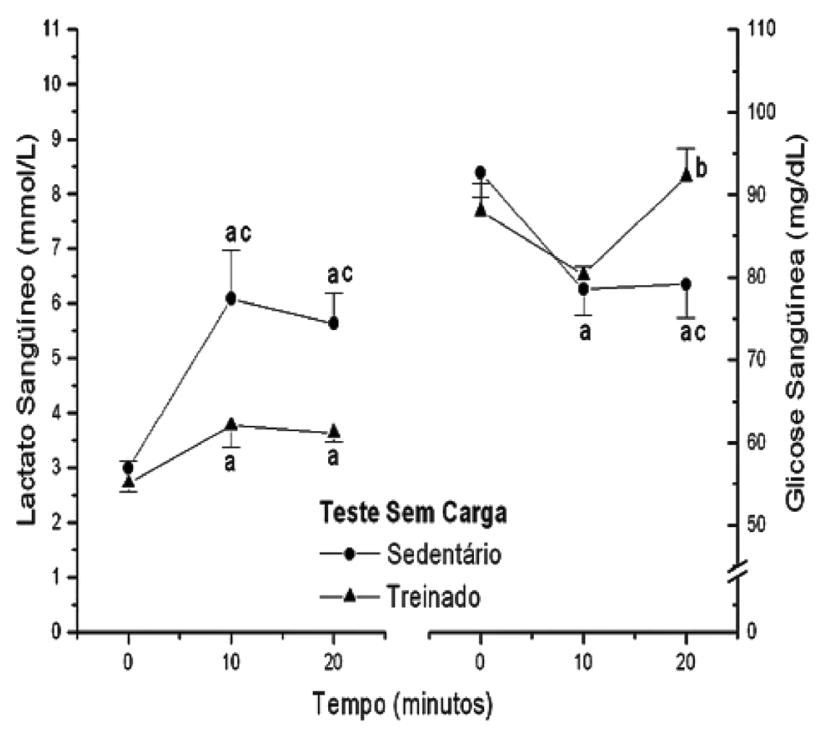

Figura 1. Concentração de lactato e glicose dos animais dos grupos sedentário e treinado $(n=6)$ durante o teste de esforço sem carga. Resultados apresentados em média \pm EPM. $a=P<0,05$ vs. tempo $0 ; b=P<0,05$ vs. tempo $10 ; c=P<0,05$ vs. mesmo tempo do treinado.

que os animais treinados conseguiram estabilizar a concentração de lactato do tempo 10 min para o tempo $20 \mathrm{~min}(5,7 \pm 0,5 \mathrm{mmol} / \mathrm{L})$, ao passo que essa estabilização nesse período $(9,2 \pm 0,9 \mathrm{~mol} / \mathrm{L} ; \mathrm{P}=0,006)$ não ocorreu nos animais sedentários.

Os resultados da glicose sanguínea dos animais sedentários mostraram redução significativa do repouso $(98,5 \pm 6,1 \mathrm{mg} / \mathrm{dL})$ para os tempos $10 \mathrm{~min}$ (carga: $77,3 \pm 6,8 \mathrm{mg} / \mathrm{dL}, \mathrm{P}=0,004)$ e $20 \mathrm{~min}(58,8 \pm 6,6 \mathrm{mg} / \mathrm{dL}$, $P<0,001)$. Porém, entre os tempos 10 e 20 min, não houve diferença. Nos treinados a glicose sanguínea manteve-se estável em todos os tempos do teste com carga (repouso: 94,0 $\pm 4,7$; tempo 10: 83,0 $\pm 3,8$ e 20 minutos: $89,8 \pm 5,7 \mathrm{mg} / \mathrm{dL} ; \mathrm{P}>0,05)$.

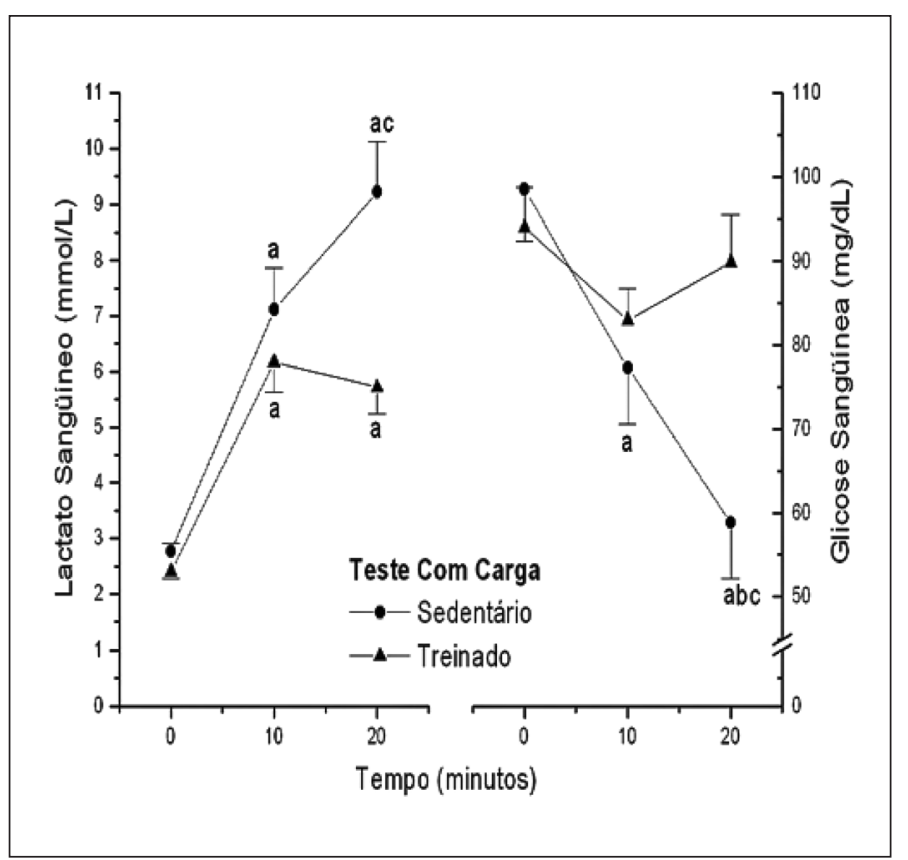

Figura 2. Concentração de lactato e glicose dos animais dos grupos sedentário e treinado $(n=6)$ durante o teste de esforço com carga. Resultados apresentados em média \pm EPM. $a=P<0,05$ vs. tempo $0 ; b=P<0,05$ vs. tempo 10; $c=P<0,05$ vs. mesmo tempo do treinado. 
Ao comparar os grupos observou-se que nos tempos repouso (sedentário: 98,5 $\pm 6,1$; treinado: 94,0 \pm 4,7mg/dL) e 10 min (sedentário: $77,3 \pm 6,8$; treinado: $83,0 \pm 3,8 \mathrm{mg} / \mathrm{dL}$ ) as concentrações não foram diferentes. Todavia, a glicose sanguínea no tempo 20 min apresentouse maior no grupo treinado do que no sedentário $(89,8 \pm 5,7$ vs. 58,8 $\pm 6,6 \mathrm{mg} / \mathrm{dL} ; \mathrm{P}=0,005)$.

A fim de verificar a associação entre as concentrações sanguíneas de lactato e glicose, foi realizado o teste de correlação de Pearson entre essas variáveis. Foi observada uma correlação negativa "forte" e significativa entre as concentrações de lactato e glicose $(P<0,001$ e $\rho=-0,742)$.

\section{DISCUSSÃO}

O objetivo deste estudo foi investigar os efeitos de um programa de treinamento aeróbio em natação com baixa intensidade (sem sobrecarga) sobre a resposta do lactato e da glicose sanguínea em ratos durante teste de esforço. Os resultados mostram que o programa de treinamento aplicado reduz a concentração de lactato e o uso da glicose durante o esforço realizado com diferentes intensidades.

O limiar anaeróbio (lactato máximo de equilíbrio), representado pelo valor máximo da estabilização entre produção e remoção do lactato sanguíneo ${ }^{(7,8)}$, é considerado um importante indicador da capacidade de exercício aeróbio e reflete a intensidade do exercício ${ }^{(14)}$. Os dados do presente estudo demonstraram que os animais sedentários atingiram essa estabilização durante o teste de esforço sem sobrecarga a uma concentração de $\sim 6 \mathrm{mmol} / \mathrm{L}$ (Figura 1), concentração semelhante à encontrada por outros ${ }^{(2,3,10)}$. Todavia, no teste com sobrecarga, os animais sedentários não foram capazes de alcançar tal estabilização. A concentração sanguínea de lactato chegou a 9,2mmol/L (Figura 2). Esses dados diferem dos apresentados por Gobatto et al.(2), onde animais sedentários da mesma linhagem apresentaram estabilização na concentração sanguínea de lactato durante exercício similar com sobrecargas entre 5 e $6 \%$ do peso corporal. Nossos resultados sugerem que a sobrecarga de $5 \%$ do peso corporal foi inadequada para que ocorresse a estabilização da concentração de lactato durante o exercício nesses animais. Provavelmente, uma carga abaixo desse nível possa ser mais adequada, conforme constatado por Voltarelli et al. ${ }^{(3)}$, os quais indicaram sobrecarga de $4 \%$ do peso corporal.

No presente estudo verificou-se estabilização na concentração de lactato dos animais treinados entre os momentos 10' e 20' para ambos os testes de esforço (Figuras 1 e 2). No entanto, essa estabilização da concentração de lactato sanguíneo no teste sem sobrecarga ocorreu em 3,8 \pm 0,4mmol/L, enquanto que no teste com sobrecarga de $5 \%$ do peso corporal ocorreu em 6,2 $\pm 0,56 \mathrm{mmol} / \mathrm{L}$. Essa redução no lactato sanguíneo durante o teste de esforço sem sobrecarga é um indicativo de que o treinamento empregado foi eficiente em melhorar a capacidade aeróbia dos animais. Isso demonstra que o treinamento alterou a cinética do lactato por meio de adaptações aeróbias, provavelmente por diminuir a produção de lactato ${ }^{(15,16)}$ e/ou aumentar sua remoção da corrente sanguínea ${ }^{(17,18)}$. Esses resultados estão de acordo com Gobatto et al. (2); entretanto, esses autores utilizaram treinamento com intensidade acima do limiar de lactato (8\% do peso corporal), em sessões de 60 minutos, durante nove semanas, ao passo que no presente estudo a intensidade foi abaixo desse limiar (sem sobrecarga), em sessões de 30 minutos, durante seis semanas. Isso sugere que a capacidade aeróbia de ratos pode ser melhorada por treinamento com intensidade abaixo do limiar de lactato e com duração menor.

Outro indicativo de que o protocolo de treinamento utilizado foi eficaz em melhorar a capacidade aeróbia dos animais treinados foi a estabilização da concentração de lactato observada durante o teste de esforço com sobrecarga (5\% do peso corporal) em $\sim 6 \mathrm{mmol} / \mathrm{L}$, concentração de estabilização alcançada pelos animais sedentários no teste sem sobrecarga. Esse resultado indica que a capacidade aeróbia dos animais melhorou, mas a concentração de lactato na qual ocorreu o equilíbrio entre produção e remoção de lactato foi mantida. Na prática, o treinamento possibilitou aos animais treinados executar uma atividade mais intensa (com sobrecarga) com a mesma concentração de lactato com a qual os animais sedentários realizaram a atividade menos intensa (sem sobrecarga) (figuras 1 e 2). Nesse sentido, Cunha et al. ${ }^{(10)}$ também mostraram que o treinamento em natação com intensidade correspondente à do limiar anaeróbio elevou a intensidade do limiar de lactato em ratos idosos.

Embora os mecanismos para explicar esse fenômeno ainda não estejam claros, parte do lactato produzido na fase inicial do exercício poderia ter sido utilizada como fonte energética na continuidade do próprio exercício, pois o treinamento utilizado foi de baixa intensidade, apresentando relativamente baixa demanda de glicose como substrato energético. Sabe-se que o lactato é um metabólito intermediário que pode ser oxidado no músculo, onde o aumento nos níveis de lactato sanguíneo poderia direcionar este para a mesma via de oxidação dos carboidratos $^{(19)}$. Miller et al. ${ }^{(20)}$ relataram que a infusão de lactato em humanos saudáveis aumentou a oxidação de lactato em detrimento da oxidação dos carboidratos, como também a maior produção de lactato pelo exercício de moderada intensidade elevou sua oxidação, poupou a glicose sanguínea e reduziu a conversão de glicogênio à glicose. Situação essa também verificada em ratos(21,22).

Segundo Messonnier et al. ${ }^{(23)}$, um mecanismo celular de tamponamento representaria a primeira linha de defesa contra a acidose e o acúmulo de lactato, podendo melhorar sua capacidade de trabalho. Esse mecanismo seria baseado em proteínas transportadoras de monocarboxilatos (MCT1 e MCT4) presentes na membrana do sarcolema e da mitocôndria, que elevariam o transporte intracelular dos prótons e lactato para o interstício durante o exercício. Thomas et al. ${ }^{(24)}$ sugerem que o treinamento aeróbio eleva o conteúdo da isoforma MCT1 no músculo esquelético, incrementando a oxidação do lactato após o exercício, por facilitar seu transporte intramuscular, como também remover o lactato sanguíneo. Py et al.(25) relatam que, em ratos, a elevação crônica do nível de lactato foi acompanhada pela alteração do seu transporte no músculo esquelético. Por outro lado, Metz et al. ${ }^{(19)}$ observaram em humanos que o maior nível de lactato não foi seguido pela alteração no transporte de lactato nas células sanguíneas, mas poderia ser afetado pelas propriedades das MCTs.

Concomitantemente à elevação da concentração sanguínea de lactato observada neste estudo (figuras 1 e 2), a concentração de glicose reduziu-se com o decorrer do exercício. Houve correlação negativa entre lactato e glicose $(P<0,001$ e $\rho=-0,742)$. Além do mais, identificouse que o teste de esforço com carga de $5 \%$ de peso corporal promoveu maior consumo de glicose sanguínea do que o teste sem sobrecarga, acarretando acúmulo de lactato sanguíneo de 9,2 mmol/L (figura 2), considerado bem acima do limiar anaeróbio para esses animais $(2,26)$. Esses resultados são similares aos de Eydoux et al.(27), que analisaram uma única série de corrida em esteira por 30 minutos numa velocidade de $25 \mathrm{~m} / \mathrm{min}$ com $10^{\circ}$ de inclinação, em ratos.

Conforme Van Meerhaeghe et al. ${ }^{(28)}$, Shulman ${ }^{(26)}$ e Denadai(4), durante o exercício intenso, existe um aumento do suprimento de ATP pela via da glicólise, em razão de a oferta de energia pela via oxidativa ser mais lenta, não suprindo a necessidade momentânea. Sob essas condições, paralelamente à elevação na produção de lactato, a taxa de liberação de prótons pela glicólise e hidrólise de ATP torna-se maior, o que pode exceder a capacidade tamponadora da célula e provo- 
car acidose muscular. Essa acidose metabólica pode desempenhar um papel determinante na intolerância ao exercício, desencadeando um declínio na força em função do prejuízo no processo excitaçãocontração, depressão da força ativada pelo $\mathrm{Ca}^{2+}$ e potencializando a fadiga muscular(23). Por outro lado, Robergs et al. (29) sugerem que a elevação do lactato é mais uma consequência do que causa da acidose metabólica.

Neste estudo verificou-se que a concentração de glicose mantevese entre os tempos 10 e 20 minutos nos animais sedentários e elevouse nos animais exercitados (Figura 1). Esse comportamento da glicose poderia ser justificado pela maior demanda energética inicial estimular a conversão do glicogênio hepático em glicose para suprir as necessidades musculares. Entretanto, devido à baixa intensidade no decorrer do exercício, o consumo de glicose como substrato energético seria reduzido, acarretando, assim, manutenção e até elevação dos níveis sanguíneos de glicose. O glicogênio muscular, ao ser convertido em glicose, é um combustível essencial para o exercício ${ }^{(11,30)}$, sendo um fator limitante ao exercício de resistência aeróbia ${ }^{(12)}$. Eydoux et al. ${ }^{(27)}$ relataram redução no glicogênio muscular entre 74 e $83 \%$ após uma corrida em esteira a $25 \mathrm{~m} / \mathrm{min}$, por 30 minutos, em ratos. Quando a demanda de energia é maior que a fonte de glicogênio muscular, entra em ação o glicogênio hepático. Contudo, esse só será liberado do fígado após ser convertido em glicose, sendo lançado na corrente sanguínea para suprir demandas elevadas nos órgãos ativos ${ }^{(26)}$.

Os resultados da concentração sanguínea de glicose para os animais treinados (Figuras 1 e 2) reforçam as afirmativas acima, onde a glicose sanguínea estabilizou e até recuperou após o tempo 10 minutos. Provavelmente, essa tendência de elevação da glicose, como também da estabilização do lactato, pode ser explicada pela substituição do substrato energético predominante utilizado durante o exercício(12,13,19). Uma das adaptações metabólicas ao exercício é a capacidade de realizar a mesma carga de trabalho reduzindo o consumo de glicose como principal substrato do exercício e, consequentemente, estabilizando o balanço de lactato. Aoki et al. ${ }^{(12)}$ descrevem uma desaceleração na taxa de utilização do glicogênio durante a realização do exercício aeróbio de longa duração. Metz et al. ${ }^{(19)}$ e Achten e Jeukendrup ${ }^{(31)}$ identificaram uma correlação inversa entre a concentração de lactato e a oxidação de lipídios como substrato energético em diabéticos, onde o aumento no lactato sanguíneo altera a mobilização de ácidos graxos livres. Estudos mostraram que o treinamento físico regula a oxidação de substratos no ciclo dos citratos e o transporte de elétrons na cadeia respiratória, elevando a capacidade oxidativa e, consequentemente, a capacidade de resistência( ${ }^{(31-33)}$. Tais mecanismos podem justificar os benefícios promovidos pelo treinamento no presente estudo, que não foram identificados nos animais sedentários (Figuras 1 e 2).

Conclui-se que o treinamento aeróbio em natação com baixa intensidade (sem sobrecarga) foi capaz de alterar as respostas do lactato e glicose sanguíneos de ratos durante os testes de esforço. O treinamento estabilizou o valor da concentração de lactato durante o exercício em níveis mais baixos do que os sedentários, em ambos os testes de esforço sem e com sobrecarga. Além disso, o treinamento promoveu adaptações na utilização do substrato energético, poupando glicose no decorrer dos testes de esforço.

Todos os autores declararam não haver qualquer potencial conflito de interesses referente a este artigo.

\section{REFERÊNCIAS}

1. Svedahl K, Maclntosh BR. Anaerobic threshold: the concept and methods of measurement. Can J Appl Physiol 2003;28:299-323.

2. Gobatto CA, de Mello MA, Sibuya CY, de Azevedo JR, dos Santos LA, Kokubun E. Maximal lactate steady state in rats submitted to swimming exercise. Comp Biochem Physiol A Mol Integr Physiol 2001;130:21-7.

3. Voltarelli FA, Gobatto CA, de Mello MA. Determination of anaerobic threshold in rats using the lactate minimum test. Braz J Med Biol Res 2002;35:1389-94.

4. Denadai BS. Limiar anaeróbio: considerações fisiológicas e metodológicas. Rev Bras Ativ Fís Saúde 1995; 1:74-88.

5. Philp A, Macdonald AL, Watt PW. Lactate: a signal coordinating cell and systemic function. J Exp Biol 2005;208:4561-75.

6. Brooks GA. Anaerobic threshold: review of the concept and directions for future research. Med Sci Sports Exerc 1985;17:22-34

7. Baldari C, Guidetti L. A simple method for individual anaerobic threshold as predictor of max lactate steady state. Med Sci Sports Exerc 2000;32:1798-802

8. Heck H, Mader A, Hess G, Mucke S, Muller R, Hollmann W. Justification of the 4-mmol/l lactate threshold. Int J Sports Med 1985;6:117-30.

9. Ascensão AA, Santos P, Magalhães J, Oliveira J, Maia J, Soares J. Concentrações sangüíneas de lactato (CSL) durante uma carga constante a uma intensidade correspondente ao limiar aeróbio-anaeróbico em jovens atletas. Rev Paul Educ Fís 2001;15:186-94.

10. Cunha VNC, Cunha RR, Segundo PR, Moreira SR, Simões HG. Treinamento de natação na intensidade do limiar anaeróbio melhora a aptidão funcional de ratos idosos. Rev Bras Med Esporte 2008;14:533-38.

11. Marliss EB, Kreisman SH, Manzon A, Halter JB, Vranic M, Nessim SJ. Gender differences in glucoregulatory responses to intense exercise. J Appl Physiol 2000;88:457-66.

12. Aoki MS, Belmonte MA, Seelaender MCL. Influência da suplementação lipídica sobre a indução do efeito poupador de glicogênio em ratos submetidos ao exercício de "endurance". Rev Paul Educ Fís 2003;17:93-103.

13. Silveira LR, Denadai BS. Efeito modulatório de diferentes intensidades de esforço sobre a via glicolítica durante o exercício contínuo e intermitente. Rev Paul Educ Fís 2002;16:186-97.

14. Chudalla R, Baerwalde S, Schneider G, Maassen N. Local and systemic effects on blood lactate concentration during exercise with small and large muscle groups. Pflugers Arch 2006;452:690-7.

15. Favier RJ, Constable SH, Chen M, Holloszy JO. Endurance exercise training reduces lactate production. J Appl Physiol 1986;61:885-9.

16. MacRae HS, Dennis SC, Bosch AN, Noakes TD. Effects of training on lactate production and removal during progressive exercise in humans. J Appl Physiol 1992;72:1649-56
17. Donovan CM, Brooks GA. Endurance training affects lactate clearance, not lactate production. Am J Physiol 1983;244:E83-92.

18. Donovan CM, Pagliassotti MJ. Enhanced efficiency of lactate removal after endurance training. J Appl Physiol 1990;68:1053-8.

19. Metz L, Sirvent P, Py G, Brun JF, Fedou C, Raynaud E, et al. Relationship between blood lactate concentration and substrate utilization during exercise in type 2 diabetic postmenopausal women. Metabolism 2005;54:1102-7.

20. Miller BF, Fattor JA, Jacobs KA, Horning MA, Navazio F, Lindinger MI, et al. Lactate and glucose interactions during rest and exercise in men: effect of exogenous lactate infusion. J Physiol 2002;544:963-75.

21. Pagano C, Granzotto M, Giaccari A, Fabris R, Serra R, Lombardi AM, et al. Lactate infusion to normal rats during hyperglycemia enhances in vivo muscle glycogen synthesis. Am J Physiol 1997;273:R2072-9.

22. Vettor R, Lombardi AM, Fabris R, Pagano C, Cusin I, Rohner-Jeanrenaud F, et al. Lactate infusion in anesthetized rats produces insulin resistance in heart and skeletal muscles. Metabolism 1997;46:684-90.

23. Messonnier L, Kristensen M, Juel C, Denis C. Importance of pH regulation and lactate/H+ transport capacity for work production during supramaximal exercise in humans. J Appl Physiol 2007;102:1936-44.

24. Thomas C, Perrey S, Lambert K, Hugon G, Mornet D, Mercier J. Monocarboxylate transporters, blood lactate removal after supramaximal exercise, and fatigue indexes in humans. J Appl Physiol 2005;98:804-9.

25. Py G, Lambert K, Perez-Martin A, Raynaud E, Prefaut C, Mercier J. Impaired sarcolemmal vesicle lactate uptake and skeletal muscle MCT1 and MCT4 expression in obese Zucker rats. Am J Physiol Endocrinol Metab 2001;281:E1308-15.

26. Shulman RG. Glycogen turnover forms lactate during exercise. Exerc Sport Sci Rev 2005;33:157-62.

27. Eydoux N, Dubouchaud H, Py G, Granier P, Prefaut C, Mercier J. Lactate transport in rat sarcolemmal vesicles after a single bout of submaximal exercise. Int J Sports Med 2000;21:393-9.

28. Van Meerhaeghe A, Velkeniers B. Lactate production and exercise-induced metabolic acidosis: guilty or not guilty? Eur Respir J 2005;26:744.

29. Robergs RA, Ghiasvand F, Parker D. Biochemistry of exercise-induced metabolic acidosis. Am J Physiol Regul Integr Comp Physiol 2004;287:R502-16.

30. Cunha TS, Tanno AP, Costa Sampaio Moura MJ, Marcondes FK. Influence of high-intensity exercise training and anabolic androgenic steroid treatment on rat tissue glycogen content. Life Sci 2005;77:1030-43.

31. Achten J, Jeukendrup AE. Relation between plasma lactate concentration and fat oxidation rates over a wide range of exercise intensities. Int J Sports Med 2004;25:32-7.

32. Knechtle B, Muller G, Willmann F, Kotteck K, Eser P, Knecht H. Fat oxidation in men and women endurance athletes in running and cycling. Int J Sports Med 2004;25:38-44.

33. Venables MC, Achten J, Jeukendrup AE. Determinants of fat oxidation during exercise in healthy men and women: a cross-sectional study. J Appl Physiol 2005;98:160-7. 